Roberto J. Beltrán Neira ${ }^{1}$ María Cristina Ikeda Artacho ${ }^{2}$

${ }^{1}$ Profesor Emérito.

Docente del Departamento Académico de Clínica Estomatológica.

Facultad de Estomatología. Universidad Peruana Cayetano Heredia.

\section{Educación en ciencias de la salud - Corrientes actuales}

Beltrán RJ, Ikeda MC. Educación en ciencias de la salud - Corrientes actuales. Rev Estomatol Herediana 2006; 16(1): 73 - 74.
En los últimos años, la educación en ciencias de la salud ha evolucionado notablemente, impulsada por nuevas tendencias en la administración de la educación que tratan de hacer más eficiente el proceso y más efectivo el resultado de la misma. Junto con los cambios tecnológicos se da la necesidad de superar el resultado deshumanizante de la educación médica centrada en la enfermedad, que ve al paciente como un objeto cuyos problemas se solucionan mediante la aplicación de tecnologías médicas o quirúrgicas. A esto se agrega el enfoque de atención al daño individual, al margen de los factores sociales y culturales propios de los enfoques preventivos de la enfermedad y promocionales de la salud, como son los de la salud pública.

La corriente dominante -y no solo en ciencias de la salud- es centrar el aprendizaje en la solución de problemas, lo cual incluye la eliminación de las asignaturas tradicionales, el contacto temprano con la comunidad y las personas con la mira puesta en atención a la salud, el logro de competencias debidamente identificadas en el perfil profesional y el diseño de este, centrado en la solución de los problemas evidenciados por la investigación.

Los procesos de acreditación internacional de programas universitarios enfatizan cada vez más la determinación y el enunciado de competencias, así como su debida incorporación por el alumno mediante procesos activos de participación personal y diálogo. También se insiste en la necesidad de disponer de formas de evaluación que cer- tifiquen el dominio de tales competencias y, en consecuencia, de los conocimientos, destrezas y actitudes que debe poseer el profesional para ser idóneo. Este enfoque de la educación exige un cambio muy importante en el profesorado, que debe hacer abandono de la tradición autoritaria aun vigente y dedicarse a investigar y aprender junto con el alumno.

Por otra parte, los recursos metodológicos de la enseñanza-aprendizaje, así como el acceso a la información, se han ampliado en gran medida con la utilización de multimedios e Internet. El dictado de clases llamadas teóricas ha cedido gran parte de su tiempo al trabajo grupal y discusión, a la búsqueda bibliográfica, a la preparación de informes y monografías, al trabajo de campo y de laboratorio, todo ello centrado en procesos destinados al desarrollo en el alumno de la actitud inquisitiva, crítica y creativa indispensable para la formación del pensamiento científico y la investigación en sus diferentes magnitudes y modalidades.

Los aspectos sociales, que anteriormente se conducían mediante actividades conocidas como de extensión o proyección social, que en numerosos casos eran prescindibles, requieren en la actualidad un contacto directo con las poblaciones en sus necesidades y aspiraciones sociales, en su contexto económico, social y político. La formación integral del nuevo profesional solo puede lograrse mediante presencia participante en la búsqueda de soluciones para los problemas de las comunidades, especialmente de las más necesitadas de atención sanitaria. En el campo de la salud, la vinculación con los servicios del estado en sus distintos niveles comenzando por la visita domiciliaria es fundamental. La vinculación temprana con los servicios periféricos y luego con el hospital debe incorporarse como parte esencial de la formación del profesional en su condición de miembro del equipo de salud al lado del médico, la enfermera y del personal auxiliar de colaboración general y específico odontológico.

La transformación de asignaturas estructuradas según el ordenamiento tradicional de las materias en módulos de aprendizaje - que tienen la virtud de integrar lo básico con lo clínico y lo social - ofrece la posibilidad de centrar mejor los aprendizajes en el logro efectivo de las competencias que se encuentren claramente identificadas en el perfil profesional del alumno al momento de su graduación.

Actualmente, la literatura en inglés muestra reiteradamente las expresiones: problem-based learning, communitybased curriculum, modular-based learning, competency-based curriculum, entre otras, como manifestaciones de la tendencia mundial a la evolución de la educación universitaria.

La experiencia de la Facultad de Estomatología de la Universidad Peruana Cayetano Heredia en investigación y desarrollo en el campo de la educación odontológica le ha permitido crear un sistema modular para la enseñanza básica, clínica y social. En la clínica se ha desarrollado el concepto de aprendizaje clínico intensivo, como actividad al- 
tamente planificada, previa al aprendizaje clínico integrado. Tanto la clínica intensiva como la clínica integral progresan a lo largo de tres niveles de complejidad creciente. Esta creación fue posible gracias a un detallado análisis de necesidades de salud y funciones odontológicas y a la construcción de una taxonomía de competencias profesionales. El modelo de clínica intensiva minimiza las denominadas preclínicas a favor de una mejor utilización del tiempo curricular, lo cual además significa un ahorro en instalaciones y equipamiento.

Los profesores de la Facultad de Estomatología de la Universidad Peruana Cayetano Heredia han contribuido a la literatura internacional sobre educación odontológica desde los primeros años de la década de 1970 hasta el presente. Sus más recientes publicaciones sobre teoría y práctica de la enseñanza de la odontología con especial referencia al aprendizaje basado en competencias aparecen al final de este artículo (1-5).

Las nuevas tendencias y sus mejores resultados operativos necesitan la formación de un equipo de docentes con disposición para desarrollar un modelo de educación que posea la originalidad que las condiciones propias de la población a la que se quiere servir demande. Aunque algunos conceptos pueden ser incorporados en discusio- nes con expertos, otros tienen que ser el producto de la reflexión personal y grupal, a la luz de una revisión amplia de la literatura en educación, especialmente en educación en medicina, odontología y enfermería. Por cierto, todo este esfuerzo tendría pocas posibilidades de alcanzar éxito de no contar con la decisión y firme apoyo político de las autoridades de la universidad.

La proliferación de facultades de odontología, orientadas muchas veces por el afán de lucro, se traduce en instituciones que repiten esquemas de enseñanza superados que mantienen y refuerzan la tendencia tecnológica y alientan la codicia en la educación del odontólogo que, como persona de servicio, debe formar parte del esfuerzo social para mejorar la salud de la población, favoreciendo la accesibilidad de las personas y comunidades a los servicios, en particular a aquellos destinados a la madre y al niño.

La práctica odontológica está cambiando a espaldas de la academia y de la profesión organizada. El nuevo enfoque para la formación del odontólogo debe prepararlo, como protagonista que es, para conducir los cambios históricos de la profesión, a fin de que se produzcan en beneficio de la salud de las personas y en respeto a las normas éticas y deontológicas que hicieron de la odontología una profesión respetable $\mathrm{y}$, del odontólogo, un hombre libre y digno para el bien común de la sociedad a la que pertenece.

\section{Referencias bibliográficas}

1. Beltrán R. Educación en Odontología - Manual del Profesor. Lima: Universidad Peruana Cayetano Heredia; 1998.

2. Beltrán RJ, Beltrán ED. Aprendizaje Intensivo a Dedicación Exclusiva. Lima: Universidad Peruana Cayetano Heredia; 2002.

3. Beltrán R, Ikeda MC. Taxonomía de Competencias en educación odontológica. Rev Estomatol Herediana 2004; 14(1-2): 107-111.

4. Beltrán RJ, Ikeda MC. Coordinación académica en la Facultad de Estomatología de la Universidad Peruana Cayetano Heredia. Rev Estomatol Herediana 2005; 15(2):187-189.

5. Bernabé E, Ludeña MA, Beltrán RJ. Self-Perceived Public Health Competency Among Recent Dental Graduates. J Dent Educa 2006; 70 (5): 571-579.

6. Ikeda MC, Beltrán RJ. Aprendizaje basado en competencias - ABC. El caso de odontología. Lima: Universidad Peruana Cayetano Heredia; 2006. 